\title{
LED Traffic Light as a Communications Device
}

\author{
Grantham Pang, Thomas Kwan, Chi-Ho Chan, Hugh Liu. \\ Dept. of Elec. \& Electronic Engineering, \\ The University of Hong Kong, \\ Pokfulam Road, Hong Kong. \\ Tel:(852)-2857-8492 \\ Fax:(852)-2559-8738 \\ Email: gpang@hkueee.hku.hk \\ URL:http://www.eee.hku.hk/ gpang
}

\begin{abstract}
The visible light from an LED (light emitting diode) traffic light can be modulated and encoded with information. Hence, it can be used for the broadcasting of audio messages or any traffic or road information. Essentially, all $L E D$ traffic lights can be used as communications devices. This paper focuses on the description of an audio information system made up of high brightness, visible light emitting diodes (LEDs) in which one or more LEDs are modulated and encoded with audio messages. The system also comprises a receiver combined with a speaker, which is located at a distance from the LED traffic light. The receiver is designed to demodulate the optically transmitted audio information and broadcast the messages with the speaker. The optical link employs intensity modulation with direct detection. The audio information system implemented on an LED traffic light can provide the function of open space, wireless broadcasting of audio messages
\end{abstract}

\section{Introduction}

Recently, high intensity light emitting diodes for traffic signals are available in the market. For example, a new bluish-green LED [1] has been designed to meet the color and brightness requirements of green traffic signals based on the standards set in the United States and other worldwide specifications. High brightness red and yellow LEDs have been in the market for some years. Hence, all three colors of high intensity, high efficiency LEDs are available to manufacturers of traffic signals. There are many advantages on the use of LEDs $[2,3,4]$. The incandescent lamps used in conventional 8-inch diameter traffic signal could consume from 116 to 150 watts of electrical power. The new LED lamp consumes only 10 to 18 watts. The life expectancy is also longer, with higher tolerance to humidity. Usually, the failure of an LED lamp is gradual, and would not cause the red, yellow or green signal to go dark suddenly. This provides an additional safety factor for motorists.

The central idea of this paper is the following. In addition to the normal function of being an indication and illumination device, an LED traffic light can be used as a communications device for the transmission and broadcasting of information. Hence, it becomes part of a wireless optical communication system. This paper describes an audio information system for the broadcasting of audio signal using visible light emitting diodes [5]. This concurrent use of visible LEDs for simultaneous signaling and communication leads to many new and interesting applications. Experimental prototypes of the system described in this paper have been implemented. The work reported in this paper differs from the use of infrared (IR) radiation as a medium for short-range wireless communications [6,7]. Currently, infrared links and localarea networks are available. IR transceivers for use as IR data links are widely available in the market at a very low cost. Comparison between the infrared and other media such as radio and microwave is given in [8]. Anyhow, there has been very little work on the use of visible light as a communication medium. The availability of high brightness LEDs also makes the visible light medium even more feasible. Essentially, all products with visible LED components (like LED traffic signal head) can be turned into an information beacon. This paper focuses on its use for audio transmission.

Visible light in fiber optics has been used as a communications medium for many years. In this paper, the usage is in the open space, as a short range information beacon. The application has to make use of the directional nature of the communication medium because the receiver requires a line-of-sight to the audio system or transmitter. In the current experimental setup, the locations of the audio signal broadcasting system and the receiver are relatively stationary. Since the relative speed between the receiver and the source are much less than speed of light, the dopplar frequency shift observed by the receiver can be safely neglected. It should be noted that the transmitter provides easy targets for the line-of-sight reception by the receiver. This is because the LEDs, being on at all time, are also indicators of the locations of the transmitter. The transmitter can broadcast with a viewing angle close to 180 degrees. 


\section{System Description}

The audio information system is shown in Figure 1. Figure 2 gives a block diagram representation of the schematic diagram of the transmitter design. The audio signal from the cassette tape or CD player has small amplitude and hence amplification of this audio signal is necessary. The audio amplifier is used to amplify the weak audio signal and to shift the average voltage level of the audio signal to an appropriate level so that the signal is within the capture range of a voltage-controlled oscillator (VCO). A VCO chip is used to modulate the incoming audio signal variations from the audio amplifier and generate the FM signal. A square wave VCO is used instead of sine wave because there are only two states (ON and OFF) for the LEDs. The carrier frequency is set at $100 \mathrm{kHz}$ with a maximum frequency deviation of $\pm 50 \mathrm{kHz}$. The modulated signal is transmitted by the switching of the LEDs. The frequency of switching is high enough such that the perceivable light appears to be constantly illuminated to the human eye.

The following is a detailed description of the receiver design as shown in Figure 3. The photo-detector circuit consists of a photodiode and a resistor. One end of the photodiode is coupled to the current limiting resistor with the other end coupled to ground. Since the signal from the photo-detector circuit is small, amplification is needed for the next stage. The limiting pre-amplifier circuit consists of two op-amplifiers as well as some resistors and diodes. The diodes are used to limit the input voltage level to a desired level (such as between $-0.7 \mathrm{~V}$ and $0.7 \mathrm{~V}$ ). This circuit aims to amplify the input signal to a certain level and a comparator is used to produce rectangular signal pulses. Two pre-amplifiers are used in this circuit because using one pre-amplifier will require a very high gain amplifier. Hence, two pre-amplifiers with lower gain each is used to achieve a high gain but with reduced noise.

Next, a data reproducing circuit is used which consists of an operational amplifier, a resistor and two NAND Schmitt triggers. The function is to produce rectangular pulses from the amplified signal in the previous stage. An operational amplifier is used as a comparator with uses virtual ground as a reference. Two NAND Schmitt trigger gates are used to enhance the noise immunity and to correct edges from low to high voltage level due to the slew rate of amplifier. Two NAND Schmitt trigger gates are used instead of one so that the signal will not be inverted. Then, a differentiator circuit consisted of a capacitor and a resistor is used to detect the leading edges of the pulse with the trailing edges blocked by a diode. Next, there is the circuit of a pulse generator. A Schmitt trigger gate is used as a pulse generator and the output gives the inverted version of pulses from the differentiator.

An integrator and envelope detector can be found in the next stage. The integrator is an envelope detector and double integrations are carried out. If the inverted pulses from the pulse generator contain high frequency, the frequency of integration is higher and the voltage level of the output would be higher. However, if the inverted pulses contain low frequency, the frequency of integration is lower and the voltage level of the output would be lower. In this way, the modulated signal would be reconstructed. Next, a band-pass filter is used. The output signal from the previous stage, integrator and envelope detector, has many distortions. A band-pass filter is used to filter out all the high frequency distortions. The higher cut-off frequency depends on a capacitor and resistor. A lower cutoff is also used to filter out the low frequency noise such as the $50 \mathrm{~Hz}$ power line frequency. The output signal from the band-pass filter is an audio signal. The final stage of the receiver circuit is a power amplifier and the output of which is connected to the speaker. The objective is for the delivery of the audible messages through a speaker or head phone/ear jack

\section{LED Traffic Light audio system}

One application of the above audio system is for LED traffic lights. Nowadays, high brightness LEDs are increasing being used in traffic lights. This is mainly due to the low power consumption and minimal maintenance required for LED-based traffic lights, which can be translated into considerable cost savings each year [9].

In the City of Philadelphia, U.S.A., the city is replacing all of its 28,000 red signals with LEDs, with an estimated annual cost savings of US 1.2 million. The next stage of development will involve the three-color LED signals. With the idea and development described in this paper, the LED traffic light can be used as a communications device, in addition to their normal function of being an indication and signaling device. This allows a concurrent use of traffic lights because it can broadcast local traffic information, vehicle location, road and navigation information, and at the same time perform its normal function of being a traffic signaling device. The LED traffic light becomes a new kind of short-range beacon to support roadside-to-vehicle communications.

\section{Implementation and Results}

Two prototypes of the audio information system described in this paper have been implemented in the Industrial Automation Laboratory at The University of Hong Kong. The first one is a simple LED transmitter consisting of HP high intensity AllnGaP LEDs with a luminous intensity of $1100 \mathrm{mcd}$ at rated $20 \mathrm{~mA}$ driving current. It is a $5 \mathrm{~mm}$ LED lamp with a 15 degree viewing angle. With just one of such LED, the authors demonstrate that the transmission distance can reach $390 \mathrm{~cm}$ with a $50 \mathrm{~mm}$ focusing lens in front of the receiver photodiode. Without the lens, the transmission distance can reach $77 \mathrm{~cm}$. Figure 4 and 5 are photographs of the implemented transmitter (with six LEDs shown) and receiver respectively.

The second implementation is an LED traffic signal head made up of 441 high brightness LEDs (Figure 6). Each has a luminous intensity of $2000 \mathrm{mcd}$ at $20 \mathrm{~mA}$ driving current, 
and the viewing angle is 30 degrees. The distance for audio transmission in outdoors can reach over $20 \mathrm{~m}$.

The radiation pattern of the LED traffic light is given in Figure 7. The frequency response characteristic of the system is shown in Figure 8. In another measurement on the signal-to-noise ratio, the result is shown in Figure 9. Figure 10 shows the distortion measurement on signals from 237 to $10 \mathrm{kHz}$.

\section{Conclusions}

LEDs provide a number of benefits in the products of many manufacturing items including long operating lifetimes, low maintenance, ruggedness, minimal heat generation, low power consumption from low current operation and compatibility with TTL and CMOS circuitry. The LED traffic lights encompass the same advantages and there is no reason for not switching to them except for the initial financing in the change. In this paper, the idea is based on the fast switching of LEDs and the modulation of the visible light. The aim is to develop an LED traffic light into a new kind of audio information system. This visible LED audio system makes use of visual light rays to transmit audio messages to a remotely located receiver. Such a system made up of high brightness visible LEDs can provide the function of open space, wireless broadcasting of audio signal. It can be used as an information beacon for short distance communication. The system is limited to line-of-sight communications. However, this concurrent use of LED traffic signal heads for simultaneous signaling and communications will open up many new applications.

\section{References}

[1] G.B. Stringfellow and M.G. Craford, "High Brightness Light Emitting Diodes" , Semiconductors and Semi-metals Vol. 48, Academic Press, 1997.

[2] M.G. Craford, "LEDs Challenge the Incandescents" , IEEE Circuits and Devices, pp. 24-29, September 1992.

[3] K. Werner, "Higher visibility for LED" , IEEE Spectrum, pp.30-39, July, 1994.

[4] P.P. Smyth, P.L. Eardley, K.T. Dalton, D.R. Wisley, P. McKee and D. Wood, "Optical Wireless - a prognosisP̈rac. On Wireless Data Transmission, SPIE Vol. 2601, pp. 212225, October 23-25, 1995

[5] E.Yang, D.Yang, G. Pang, C.Yeung, S.W. Cheung, M.Hong, P. Kwok, K.W. Tse, L.Ko and T. Kwan, "Audio Information system using light emitting diodes" , Provisional US patent application.

[6] T.S. Chu and M.J. Gans, "High speed infrared local wireless comm." , IEEE Communications Magazine, pp. 4-10, August 1997.

[7] M. Meyer, "Infrared LEDs" , Compound Semiconductor, pp. 39-40, May/June, 1996.

[8] J.M. Kahn and J.R. Barry, "Wireless Infrared Communications" , Proceedings of the IEEE, Vol. 85, No.2, pp.265-298, Feb 1997.

[9] J. O' Connell, “The Philadelphia story” , Traffic Technology International, UK \& International Press, pp. 106-110, Aug/Sept. 1997. 


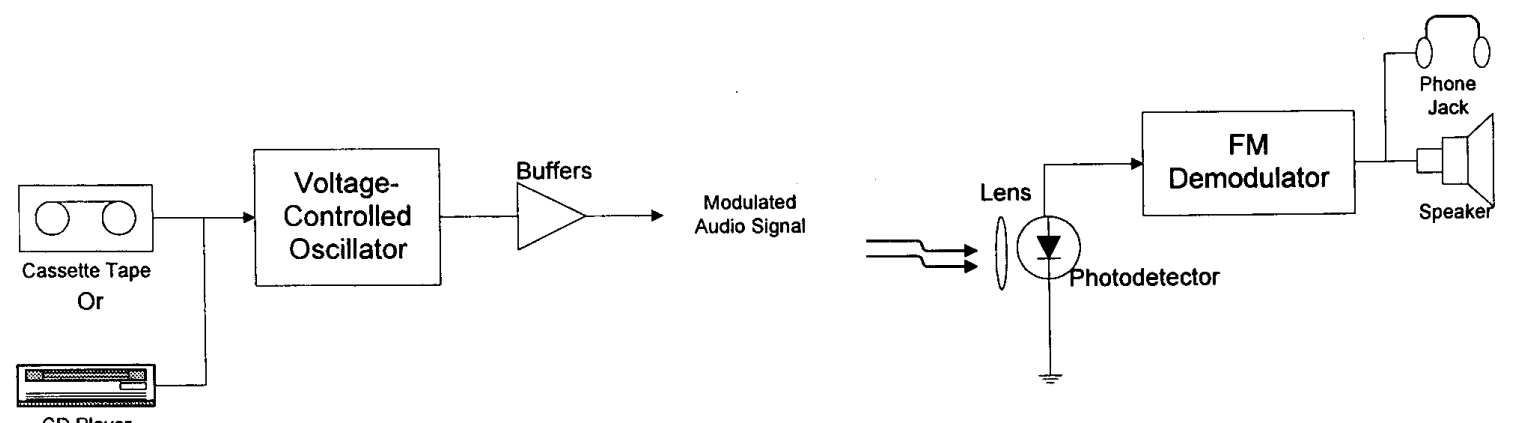

Fig. 1 Block diagram of the audio information system, which includes a transmitter and a companion receiver.

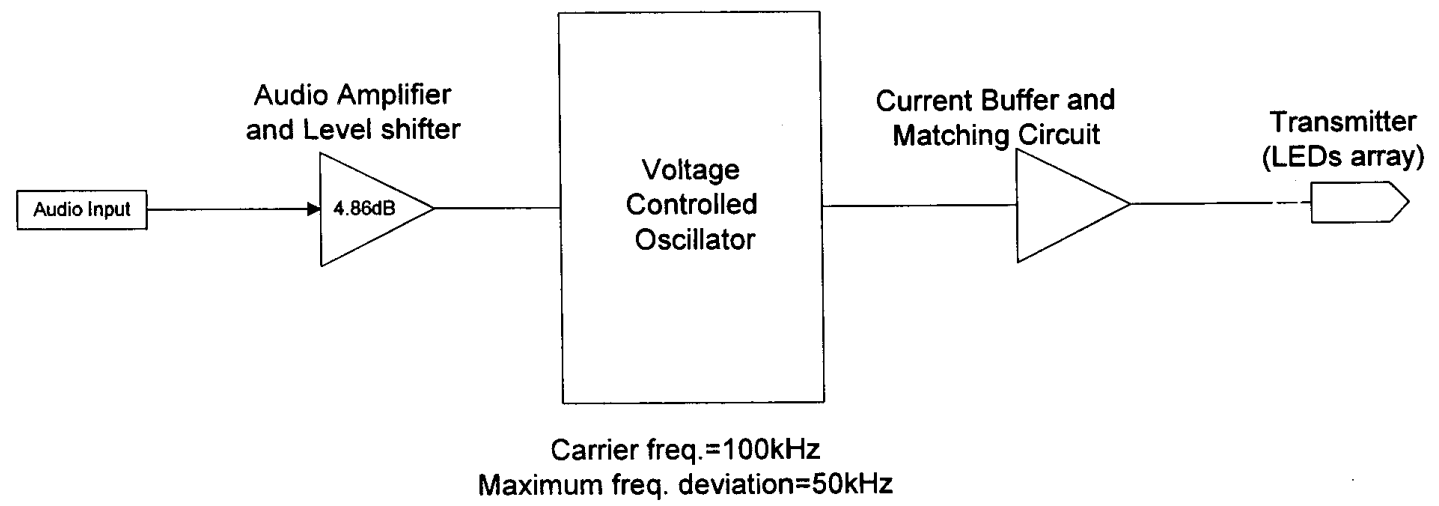

Fig. 2 Block diagram representation of the schematic diagram of the transmitter design.



Fig. 3 Block diagram representation of the schematic diagram of the receiver design. 


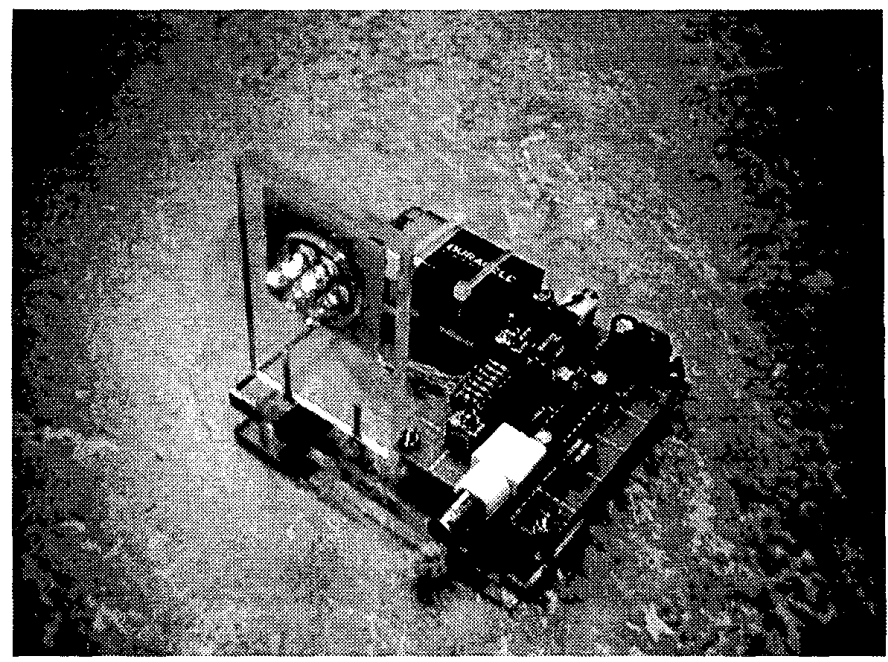

Fig. 4 Photograph of the transmitter.

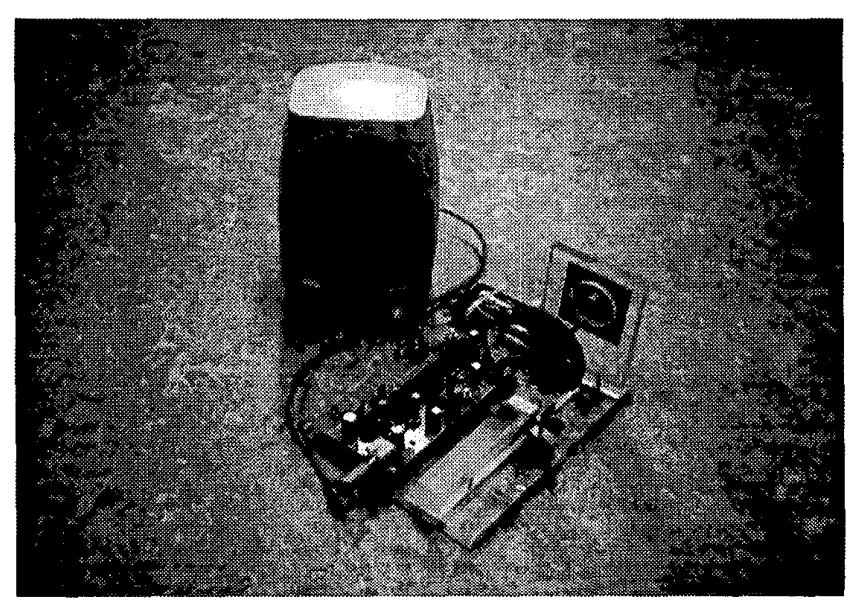

Fig. 5 Photograph of the receiver.



Fig. 6 Photograph of the LED traffic light. 


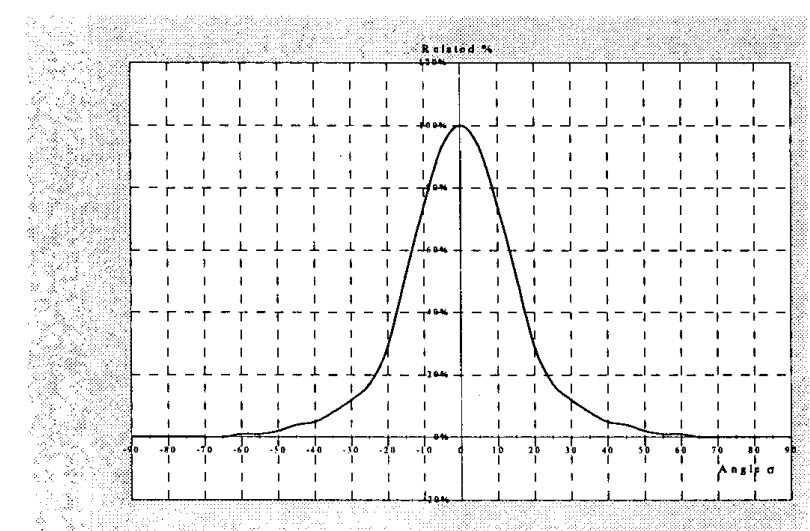

Fig 7. Radiation pattern of the LED traffic light.


Fig 8. Frequency response characteristics of the system.

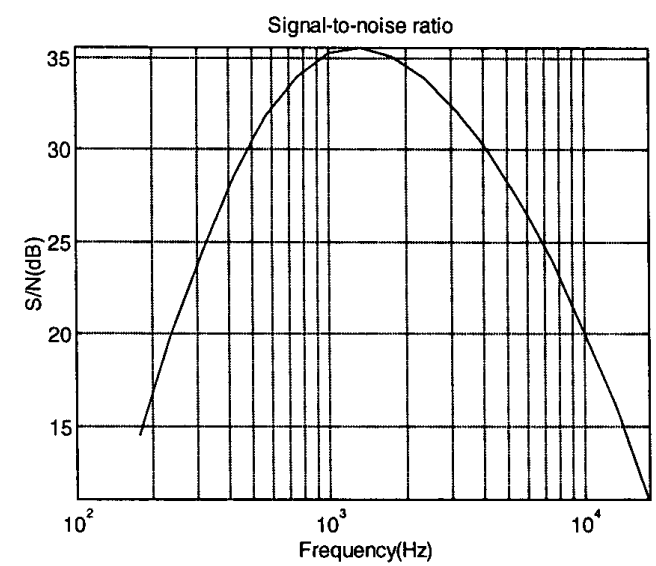

Fig.9. Measurement on the signal-to-noise ratio

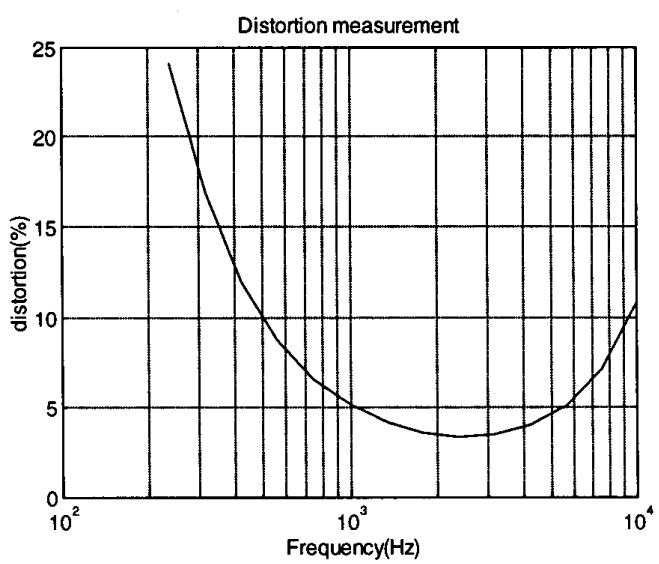

Fig.10. Distortion measurement of the system 